\title{
Thermal Imaging And Its Application In Defence Systems
}

\author{
Aparna Akula, Ripul Ghosh, H K Sardana \\ Academy of Scientific and Innovative Research (AcSIR) \\ Central Scientific Instruments Organisation, Chandigarh -160030, India \\ aparna.akula@csio.res.in,ripul.ghosh@csio.res.in
}

\begin{abstract}
Thermal imaging is a boon to the armed forces namely army, navy and airforce because of its day night working capability and ability to perform well in all weather conditions. Thermal detectors capture the infrared radiation emitted by all objects above absolute zero temperature. The temperature variations of the captured scene are represented as a thermogram. With the advent of infrared detector technology, the bulky cooled thermal detectors having moving parts and demanding cryogenic temperatures have transformed into small and less expensive uncooled microbolometers having no moving parts, thereby making systems more rugged requiring less maintenance. Thermal imaging due to its various advantages has a large number of applications in military and defence. It is popularly used by the army and navy for border surveillance and law enforcement. It is also used in ship collision avoidance and guidance systems. In the aviation industry it has greatly mitigated the risks of flying in low light and night conditions. They are widely used in military aviation to identify, locate and target the enemy forces. Recently, they are also being incorporated in civil aviation for health monitoring of aircrafts.
\end{abstract}

Keywords: Thermogram, Infrared, FLIR, Motion Detection, ATR

PACS: 89.20.Dd, 42.30.Va, 42.79.Ls

\section{INTRODUCTION}

In the late 1960's real-time thermal imaging technology started becoming prominent. An infrared thermal imaging camera is one which can produce images of invisible infrared energy. Infrared energy is part of the electromagnetic spectrum, which we perceive as heat and is invisible to the naked eye. Some level of thermal energy is emitted from all living, objects, and materials. Infrared technology can be used to capture this energy and represent it in the form of a thermogram which can be perceived as images. Thermal imaging can successfully penetrate such environments as smoke, light fog, snow, rain and extreme darkness. They are also capable of scanning broad areas and are a good choice to use aboard aircrafts or in satellite devices.

Some of the greatest advances in infrared technology have occurred in defence systems. One such advancement is Forward Looking Infrared, or $F L I R$, technology which originated as a sensor system for fighter aircraft. FLIR is a forward sensor because it is fixed mainly to view what is directly in front of the aircraft. It is also a forward sensor in the sense that the image is displayed to the pilot through the aircraft's heads-up display (HUD). Predecessor FLIR technology was originally developed by the United States Navy to identify and target enemy forces. These systems were large, expensive and heavy. With the evolution of advanced technology rugged and more efficient FLIR cameras with reduced size and prices became available [1].

The former part of the paper describes briefly the principle of thermal imaging and the detector technology. This is followed by a brief detail of widely spanning applications of thermal imaging in the defence industry. Lastly, the paper describes the algorithm for detection of moving targets in thermal imagery.

\section{PRINCIPLE OF THERMAL IMAGING}

Infrared radiation occupying the portion of electromagnetic spectrum in the band of $0.9-14 \mu \mathrm{m}$ is emitted by all objects at temperatures above absolute zero. IR camera represents the captured radiation as a thermogram, a gray or pseudo colour thermal image that depicts thermal variations across an object or scene. IR cameras are based on two laws, the total radiation law and the Stefan - Boltzmann's law. Total radiation law states that the amount of incident energy is equal to the sum of absorbed, reflected and

Optics: Phenomena, Materials, Devices, and Characterization

AIP Conf. Proc. 1391, 333-335 (2011); doi: 10.1063/1.3643540

(C) 2011 American Institute of Physics 978-0-7354-0960-6/\$30.00 
transmitted energy. Stefan - Boltzmann's law takes the form,

$$
W=\varepsilon \sigma T^{4}
$$

which states that the total radiant energy $(W)$ of a body is proportional to the emissivity $(\varepsilon)$ and to the fourth power of temperature $(T)$.

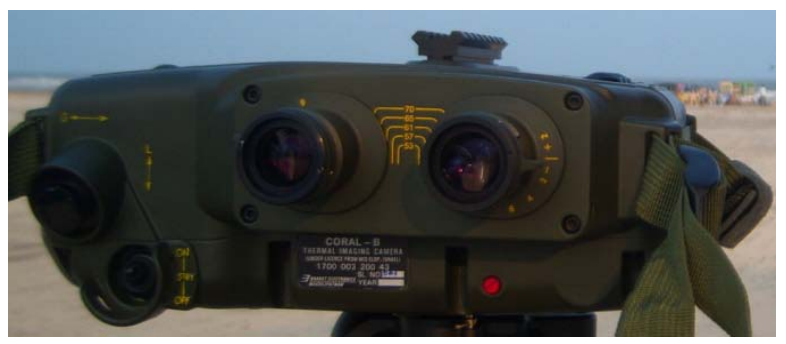

FIGURE 1. Coral -B, Uncooled microbolometer type handheld thermal Imager

Thermal imagers are analogous to digital cameras except that charge coupled devices (CCD) or CMOS detectors of digital cameras are replaced by focal plane array (FPA) of micrometer size pixels of various materials which are sensitive to IR radiation. Enhancements in IR cameras are mainly due to the technological advancement of detectors, which has transitioned from point (single) detector to linear and array (FPA) detectors. FPA technologies are of two types, thermal and quantum detectors. Quantum detectors are made from materials such as InSb, InGaAs, PtSi, HgCdTe (MCT), and layered GaAs/AlGaAs for QWIP (Quantum Well Infrared Photon) detectors. Uncooled microbolometer is a common type of thermal detector made of a metal or semiconductor material sensitive to temperature. A commercial thermal camera is shown in Fig. 1. Thermal detectors typically have lower cost and a broader IR spectral response than quantum detectors. However, quantum detectors are generally faster and more sensitive than thermal detectors but they require cooling, down to cryogenic temperatures [2].

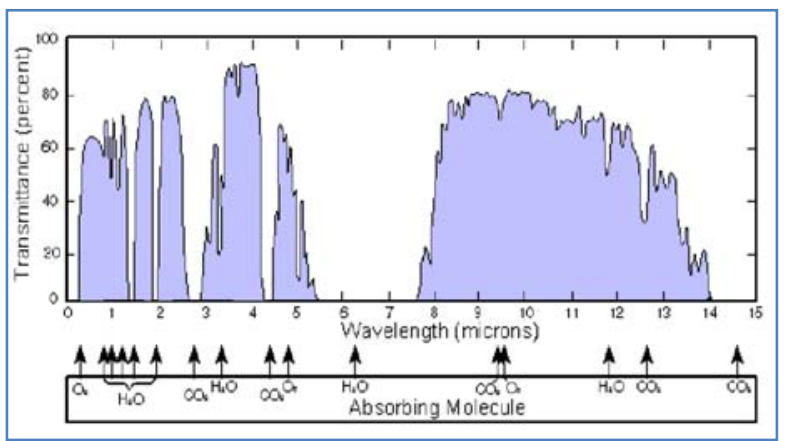

FIGURE 2. Atmospheric attenuation in the IR region.
Thermal imaging systems are designed to operate at two wavelength windows, mid-wave infrared (MWIR) band of 3 to 5 microns and long-wave infrared (LWIR) band of 8 to 12 microns. Atmospheric attenuation mostly due to water vapour is maximum in MWIR band and hence not used for imaging. LWIR band is least susceptible for atmospheric attenuation and hence widely used in harsh environments. Atmospheric attenuation in the infrared region is shown in Fig. 2 [3].

\section{APPLICATIONS}

Thermal energy emerged as an imaging technology in the years just following World War II. Its spectrum consist of diverse domains like military, navy, aviation, automotive, geological survey, agriculture, fault detection and many more. Thermal imaging systems are widely in use for military use. With the reducing prices of IR technology their use for civilian surveillance applications is considered widely. They are used in industry to detect serious faults in equipment, process control and quality control.

One of the main uses of thermal imaging is in the aviation industry, mainly for military purposes. Fighter aircraft Pilots look through the Head up Display (HUD) with the FLIR up and attempt to locate and identify the target [4]. FLIR system reduces the risk of low light and visibility conditions. FLIR-assisted landing has proven to be a great success with reduced number of night landing accidents. FLIR when combined with the radar sensors are a potential component of an airborne monitoring system for locating an active runway as well as nearby ground traffic when normal visibility is reduced by meteorological conditions or darkness. It also forms an essential tool for aircraft health monitoring.

FLIR cameras are widely used in automatic target detection and designation systems. These systems mainly consist of two parts, navigation pod and targeting pod. The navigation pod generally is fitted with a wide field of view FLIR camera and a terrain following radar (TFR) system, which enables accurate low-level penetration under all weather conditions. The targeting pod consists of a narrow field of view FLIR camera and laser ranger/designator [5].

Thermal imaging is widely used in airborne surveillance systems of spy planes which are used to monitor enemy forces, as well as monitor other suspicious activities using satellite imaging. It is also largely used in enhanced satellite imaging to monitor storm activity and environmental patterns which forms the basis for prediction models. Law enforcement agencies also use this technology to trace criminals. 


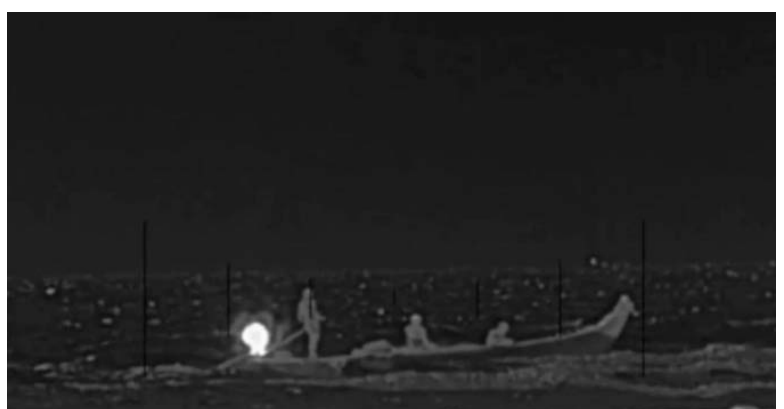

FIGURE 4. Coastal area surveillance

Thermal imaging is widely used by the naval systems for navigation purposes to avoid ship collision during night and for monitoring the coastal areas to curb any unlawful activities. A typical example of coastal surveillance is shown in Fig. 5, thermogram of a motor boat approaching the shore during night.

\section{MOTION DETECTION}

Motion detection is an important task of Automatic Target Recognition (ATR) and perimeter monitoring systems. ATR is the field of science to detect, track, classify targets from video and signal streams. Perimeter or border monitoring mainly consists of detecting any unwanted or unexpected movement of person or vehicles in and around the area under surveillance. Thermal imaging has become an integral part of these systems because of its ability to operate in all weather conditions.

Typical moving target identification (MTI) system consists of a thermal imager, frame grabber, processing system, communication system and software comprising of algorithms to detect motion in the thermal images. For long range surveillance applications generally the images containing the moving target are transmitted to the base station for manual interpretation. In case of short range surveillance, the MTI systems are made intelligent to recognize if the moving target is a potential enemy target and transmits this information to a firing system. Block diagram representing the various stages of a typical ATR/MTI system is shown in Fig. 5.

Motion detection algorithm was implemented in MATLAB environment. The initial frame containing no object is taken as background image. The background is subtracted from the current frame to obtain an image containing motion information. The image is processed to remove the clutter and enhance the quality of target for further processing. Thermal image sequences containing moving vehicles with a static background is used to test the algorithm. The results obtained for a test sequence containing a vehicle in motion is shown in Fig. 6. Better results can be obtained by dynamic modeling of background.

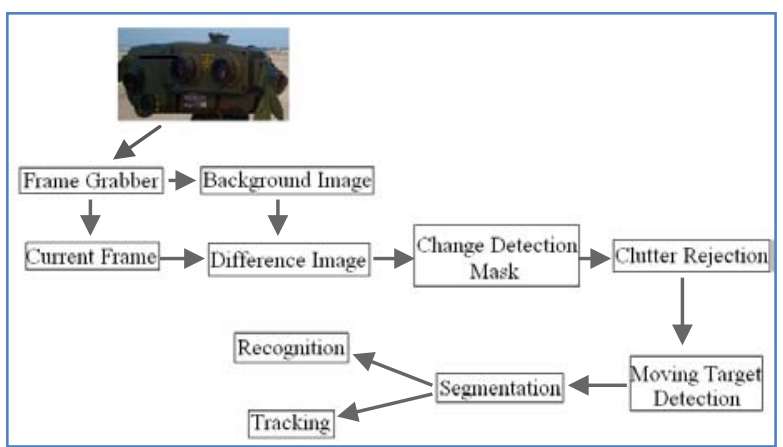

FIGURE 5. Block diagram of motion detection (a) Input Image

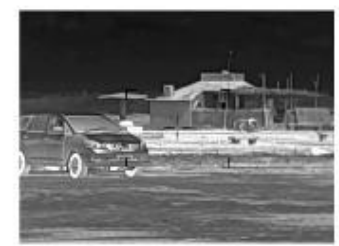

(c) Before Processing

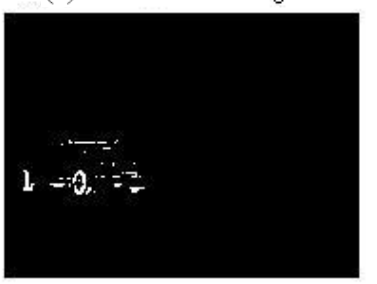

(b) Background Image

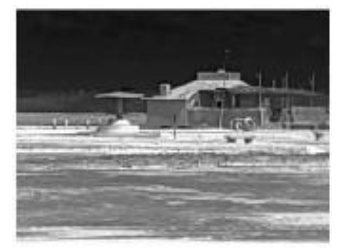

(d) Foreground Image

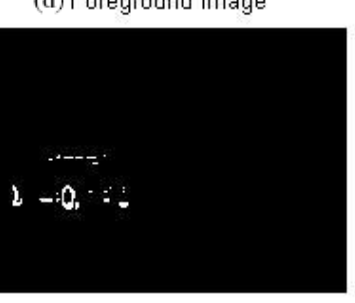

FIGURE 6. (a) Current frame, (b) Background Image, (c) Output of change detection stage, (d) Output of clutter rejection stage

\section{ACKNOWLEDGEMENTS}

The authors wish to acknowledge Vinod Karar, Satish Kumar of Central Scientific Instruments Organisation, Chandigarh and Dr. S S Neggi and team of Thermal Imaging Laboratory, Instruments Research and Development Establishment, Dehradun, for their valuable inputs. The authors are thankful to Bharat Electronics Limited for providing the thermal imager.

\section{REFERENCES}

1. Gary P. Moynihan, Joseph Thomas, F-16 Risk Analysis: Block 60 FLIR-Assisted Landing Instruction, Air Force Journal of Logistics, Volume XXX, No. 3, Fall, 2006.

2. FLIR systems, The Ultimate Infrared Handbook for R\&D Professionals.

3. http://www.thefullwiki.org/Infrared

4. Robert B. Wood, Peter J. Howells, Head Up Displays, Chapter 4, The Avionics Handbook.

5. Carlo Kopp, Laser Guidance, Australian Aviation and Defence Review, September, 1981. 